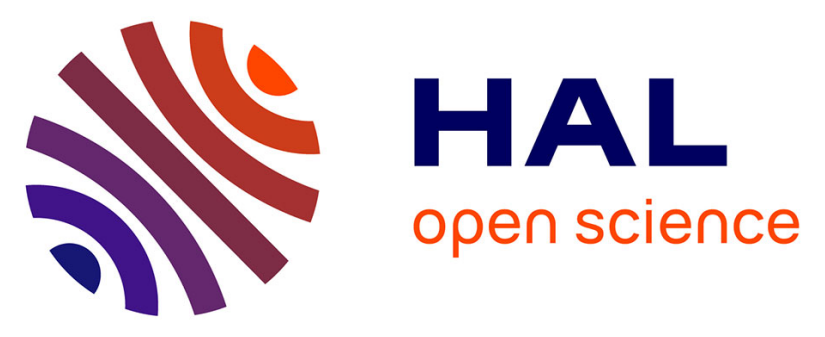

\title{
A Time-Domain finite element homogenization technique for lamination stacks using skin effect sub-basis functions
}

Patrick Dular, Johan Gyselinck, Laurent Krähenbühl

\section{- To cite this version:}

Patrick Dular, Johan Gyselinck, Laurent Krähenbühl. A Time-Domain finite element homogenization technique for lamination stacks using skin effect sub-basis functions. COMPEL: The International Journal for Computation and Mathematics in Electrical and Electronic Engineering, 2006, 25 (1), pp.6-16. 10.1108/03321640610634281 . hal-00082847

\section{HAL Id: hal-00082847 https://hal.science/hal-00082847}

Submitted on 16 Mar 2007

HAL is a multi-disciplinary open access archive for the deposit and dissemination of scientific research documents, whether they are published or not. The documents may come from teaching and research institutions in France or abroad, or from public or private research centers.
L'archive ouverte pluridisciplinaire $\mathbf{H A L}$, est destinée au dépôt et à la diffusion de documents scientifiques de niveau recherche, publiés ou non, émanant des établissements d'enseignement et de recherche français ou étrangers, des laboratoires publics ou privés. 


\title{
A Time-Domain finite element homogenization technique for lamination stacks using skin effect sub-basis functions
}

\author{
P. Dular ${ }^{1}$, J. Gyselinck ${ }^{2}$, L. Krähenbühl ${ }^{3}$ \\ ${ }^{1}$ University of Liège - Dept. of Electrical Engineering and Computer Science - Inst. Montefiore B28 - B-4000 Liège - Belgium \\ 2 Free University of Brussels (ULB), Dept. of Electrical Engineering, B-1050 Brussels, Belgium \\ ${ }^{3}$ CEGELY (UMR CNRS 5005) - École Centrale de Lyon - F-69134 Écully Cedex - France
}

Purpose - Development of an homogenization technique to directly and efficiently take the eddy current effects in laminated magnetic cores within time domain finite element (FE) analyses.

Design/methodology/approach - The technique is developed for being used within a $3 \mathrm{D}$ magnetodynamic $\mathbf{b}$-conform $\mathrm{FE}$ formulation, e.g., using a magnetic vector potential. To avoid a fine FE discretization of all the laminations of a magnetic core, this one is considered as a source region that carries predefined current and magnetic flux density distributions describing the eddy currents and skin effect along each lamination thickness. Both these distributions are related and are first approximated with sub-basis functions. Through the homogenization or averaging of the sub-basis functions contributions in the FE formulation, the stacked laminations are then converted into continuums, thus implicitly considering the eddy current loops produced by parallel magnetic fluxes. The continuum is then approximated with classical FE basis functions and can be defined on a coarser discretization.

Findings - The developed method appears attractive for directly and efficiently taking into account within finite element analyses the eddy current effects, i.e., the associated losses and magnetic flux reduction, that are particularly significant for high frequency excitations. The time domain analysis allows the consideration of both nonlinear and transient phenomena.

Originality/value - The averaging of sub-basis functions contributions, describing fine distributions of fields, in a FE formulation leads to an original way to homogenize laminated regions. The proposed method is naturally adapted for time domain analyses and in some sense generalizes what can be done more easily in the frequency domain.

Keywords - Eddy currents, Finite element analysis, Homogenization, Lamination stack

Paper type - Research paper

\section{INTRODUCTION}

Iron cores in electrical devices are usually made of lamination stacks in order to reduce the eddy current losses due to time-varying flux excitations. When simulating such devices using the finite element (FE) method, it is usually impossible to model the eddy currents in each separate lamination. Commonly these currents are first completely ignored, whereupon the Joule losses may be estimated from the results of the eddy current free model (Bertotti et al., 1991). This nevertheless leads to neglect the flux reduction due to the eddy currents, while this reduction can be significant for high frequency excitations.

A technique is proposed here to directly take these losses into account with a b-conform 3-D FE formulation using the magnetic vector potential. The technique is a time-domain extension, in particular to allow nonlinear analyses, of the frequency domain method proposed by Dular et al. (2003)

This work was supported by the Belgian Science Policy (IAP P5/34) and the Belgian French Community (Research Concerted Action ARC 03/08-298).

P. Dular is a Research Associate with the Belgian National Fund for Scientific Research (F.N.R.S.) and Krähenbühl et al. (2004) and an alternative to the method proposed by Gyselinck and Dular (2004) for homogenizing the laminations in a formulation. It also generalizes the method proposed by Righi et al. (2001). It consists in considering the lamination stack as an homogenized source region carrying predefined current density distributions, as well as the associated magnetic flux density distributions, describing the eddy currents and skin effect in each lamination. The expressions of such related current and magnetic flux densities and their use in the magnetic vector potential formulation constitute the key objective of this paper. Some results are illustrated and compared for test problems.

\section{B-CONFORM FE FORMULATION AND LAMINATION STACKS}

A bounded domain $\Omega$ of the three-dimensional Euclidean space is considered, in which the magnetodynamic problem is defined. Massive conductors, subject to eddy currents, belong to $\Omega_{\mathrm{c}} \subset \Omega$ and source conductors, carrying a given current density $\mathbf{j}_{\mathrm{s}}$, are regions of $\Omega_{\mathrm{s}} \subset \Omega$.

With the $\mathbf{b}$-conform magnetic vector potential formulation, the general expression of the electric field e via a magnetic vector potential a involves the gradient of an electric scalar potential $\mathrm{v}$ in $\Omega_{\mathrm{c}}$, i.e.,

$$
\mathbf{e}=-\partial_{\mathrm{t}} \mathbf{a}-\operatorname{grad} \mathbf{v} \text { in } \Omega_{\mathrm{c}}
$$

with

$$
\mathbf{b}=\operatorname{curl} \mathbf{a} \text { in } \Omega \text {, }
$$

so that the Faraday equation is satisfied; $\mathbf{b}$ is the magnetic flux density and $\mathrm{v}$ can usually be fixed to zero in passive conductors (not supplied by external sources). With these two potentials, the a-v magnetodynamic formulation is obtained from the weak form of the Ampere equation, i.e. (Dular et al., 2003),

$$
\begin{aligned}
& \left(\mu^{-1} \operatorname{curl} \mathbf{a}, \operatorname{curl} \mathbf{a}^{\prime}\right)_{\Omega}+\left(\sigma \partial_{\mathrm{t}} \mathbf{a}, \mathbf{a}^{\prime}\right)_{\Omega_{\mathrm{c}}}+\left(\sigma \operatorname{grad} \mathrm{v}, \mathbf{a}^{\prime}\right)_{\Omega_{\mathrm{c}}} \\
& -\left(\mathbf{j}_{\mathrm{s}}, \mathbf{a}^{\prime}\right)_{\Omega_{\mathrm{s}}}=0, \forall \mathbf{a}^{\prime} \in \mathrm{F}_{\mathrm{a}}(\Omega),
\end{aligned}
$$

where $\mu$ is the magnetic permeability, $\sigma$ is the electric conductivity, $\mathrm{F}_{\mathrm{a}}(\Omega)$ is the function space defined on $\Omega$ and containing the basis functions for a and test function $\mathbf{a}^{\prime} ;(\cdot,)_{\Omega}$ denotes a volume integral in $\Omega$ of the product of its vector field arguments.

The direct way to consider the eddy currents in a lamination stack is to model this stack as a set of massive conductors (i.e., a subset of $\Omega_{\mathrm{c}}$ ) separated by insulating layers. This is nevertheless generally unfeasible in view of the large number of laminations encountered in iron cores. An alternative method consists in considering the lamination stack as an homogenized region (i.e., rather a subset of $\Omega_{\mathrm{s}}$ ) through 
predefined current density and magnetic flux density distributions in each lamination. These distributions can be expressed in terms of adequate basis functions and unknown associated coefficients.

\section{PREDEFINED EDDY CURRENT AND MAGNETIC FLUX DENSITY DISTRIBUTIONS IN A LAMINATION STACK}

\section{A. Eddy current density versus the magnetic flux density}

A lamination stack region $\Omega_{1 \mathrm{~s}} \subset \Omega$ is considered (Fig. 1). Each lamination has a thickness $\mathrm{d}$, an electric conductivity $\sigma$ and a magnetic permeability $\mu$. It is described by a local coordinate system $\left(\mathbf{i}_{\alpha}, \mathbf{i}_{\beta}, \mathbf{i}_{\gamma}\right)$, for which the directions $\mathbf{i}_{\alpha}$ and $\mathbf{i}_{\beta}$ are parallel to the lamination plane, while $\mathbf{i}_{\gamma}$ is perpendicular to it. The direction $\mathbf{i}_{\alpha}$ is considered as the a priori unknown direction of the magnetic flux density $\mathbf{b}_{\alpha}$ parallel to the lamination, and consequently $\mathbf{i}_{\beta}$ is the main direction of the eddy current loops generated by variations of $\mathbf{b}_{\alpha}$, with the associated current density $\mathbf{j}_{\beta}$.

The effect of a varying magnetic flux density perpendicular to the laminations can be taken into account in (3) through an anisotropic conductivity having a zero component along $\mathbf{i}_{\gamma}$. Its consideration in the developed formulation would lead to non-zero net currents for $\mathbf{j}_{\beta}$.

The current density $\mathbf{j}_{\beta}$ has to undergo a pretreatment for avoiding, at the discrete level, the discretization of each lamination separately. This pretreatment concerns the strongly expressed equations in the considered formulation, i.e., the Faraday and the flux conservation equations for $\mathbf{a} \mathbf{b}$ conform formulation, while the Ampere equation is rather kept in a weak form.

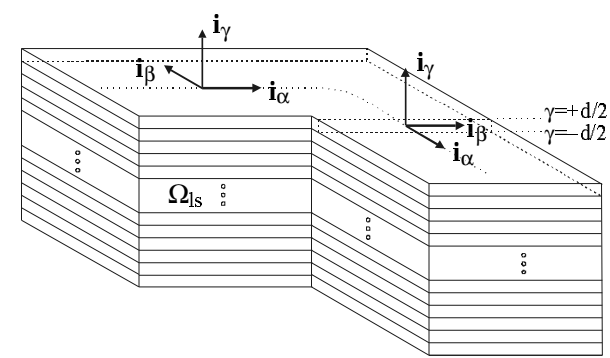

Fig. 1. Lamination stack $\Omega_{\text {ls }}$ with its local coordinate system $\left(\mathbf{i}_{\alpha}, \mathbf{i}_{\beta}, \mathbf{i}_{\gamma}\right)$ associated with each lamination.

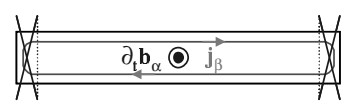

Fig. 2. Magnetic flux density $\mathbf{b}_{\alpha}$ and associated current density $\mathbf{j}_{\beta}$ in the cross section $\left(\mathbf{i}_{\beta}, \mathbf{i}_{\gamma}\right)$ of a lamination stack $\Omega_{1 s}$; fringing effects are neglected.

By the 1-D Faraday equation neglecting fringing effects of $\mathbf{j}_{\beta}$ (Fig. 2), one has for one lamination, with thickness coordinate $\gamma$,

$$
\partial_{\gamma} \mathbf{e}_{\beta}=\mathbf{i}_{\gamma} \times \partial_{\mathrm{t}} \mathbf{b}_{\alpha}
$$

where $\mathbf{e}_{\beta}$ is the $\beta$-directed electric field. Taking the primitive of (4) and the Ohm law, the current density $\mathbf{j}_{\beta}$ can be expressed in terms of a primitive in $\gamma$ of $\mathbf{b}_{\alpha}$, i.e.

$$
\mathbf{j}_{\beta}=\sigma \mathbf{e}_{\beta}=\sigma \mathbf{i}_{\gamma} \times \partial_{\mathrm{t}} \int \mathbf{b}_{\alpha} \mathrm{d} \gamma .
$$

\section{B. Magnetic flux density approximate expansion}

Field $\mathbf{b}_{\alpha}$ in (5) is unknown and is given along the thickness of each lamination a predefined approximate expansion limited to a certain order $p$, i.e. for its component $b_{\alpha}$, with $\mathbf{b}_{\alpha}=b_{\alpha} \mathbf{i}_{\alpha}$

$$
b_{\alpha}(\gamma, t)=\sum_{n \in S} b_{n}(t) \eta_{n}(\gamma),
$$

where functions $\eta_{n}(\gamma)$ define sub-basis functions for $\mathbf{b}_{\alpha}$, that differ from the constant function because of the skin effect for this field, and $\mathrm{S}$ defines a set of expansion orders. A constant $\mathbf{b}_{\alpha}$ has been considered by Righi et al. (2001) and Dular et al. (2003) for a low frequency model. A higher approximation order is now intended to be defined.

The so-called skin effect sub-basis functions can be freely chosen as polynomials (Gyselinck and Dular, 2004) or as more sophisticated expressions closer to the expected actual distribution of $\mathbf{b}_{\alpha}$ (e.g., hyperbolic functions). In case no net current exists in the laminations, even polynomial basis functions can be considered.

The purpose of expansion (6) is to avoid the definition of a FE mesh of each lamination approximating accurately enough the skin effect. Here, this distribution will be rather considered by a pretreatment of sub-basis functions in the FE formulation, as will be shown, instead of using explicit classical basis functions of a sufficient number of thin FEs along the lamination thickness.

Coefficients $b_{n}, n \in S$, are peculiar to each lamination. For the whole lamination stack, they will be converted to a continuum, or homogenized, i.e. continuously varying from one lamination to the next. This means that other basis functions are needed for the approximation of this continuum in direction $\mathbf{i}_{\gamma}$; a variation along the longitudinal direction $\mathbf{i}_{\alpha}$ should be allowed as well. These basis functions will be associated with the so-defined homogenized FE mesh.

For a b-conform formulation, the strong expression of the flux conservation, i.e., $\operatorname{div} \mathbf{b}=0$, has to be applied to (6) for giving particular properties to coefficients $b_{n}$. An adequate choice for the sub-basis functions will be shown to simplify, among other things, the expression of such properties

\section{HOMOGENIZATION PROCESS IN THE MAGNETIC VECTOR POTENTIAL FE FORMULATION}

The lamination stack is considered as a source region (i.e., in $\Omega_{\mathrm{s}}$, rather than a set of massive conductors, in $\Omega_{\mathrm{c}}$ ) carrying the predefined current density (5) in place of source current $\mathbf{j}_{\mathrm{s}}$ in (3), as well as the associated predefined magnetic flux density $\mathbf{b}_{\alpha}$.

Both first and fourth terms in (3) have to give contributions similar to these given by a massive conductor region (Dular et al., 2003), expressing the weak form of the Ampere equation applied to expressions (5) and (6) for $\mathbf{j}_{\beta}$ and $\mathbf{b}_{\alpha}$ (these discrete expressions exactly satisfying the Faraday equation can indeed only be related in a weak way in the Ampere equation). This is achieved by using (5), (6), as well as $\mathbf{e}_{\beta}=-\partial_{\mathrm{t}} \mathbf{a}_{\beta}$ leading to $\mathbf{a}_{\beta}=-\mathbf{i}_{\gamma} \times \int \mathbf{b}_{\alpha} \mathrm{d} \gamma$. The associated test functions have to be considered as well.

One has, for the first term of (3),

$$
\begin{aligned}
& \left(\mu^{-1} \text { curla,curla' }\right)_{\Omega_{\mathrm{ls}}}=\sum_{\mathrm{i}}\left(\mu^{-1} \mathbf{b}_{\alpha}, \mathbf{b}_{\alpha}{ }^{\prime}\right)_{\Omega_{\mathrm{l}, \mathrm{i}}} \\
& =\sum_{\mathrm{i}}\left(\mu^{-1} \sum_{\mathrm{n}} \mathrm{b}_{\mathrm{n}} \eta_{\mathrm{n}}, \sum_{\mathrm{m}} \mathrm{b}_{\mathrm{m}}{ }^{\prime} \eta_{\mathrm{m}}\right)_{\Omega_{\mathrm{ls}, \mathrm{i}}}
\end{aligned}
$$

where the contribution of $\Omega_{1 \mathrm{~s}}$ is split up into a sum of contributions of each of its laminations $\Omega_{1 \mathrm{~s}, \mathrm{i}}$ (i.e., the i-th lamination in $\Omega_{1 \mathrm{~s}}$ ). Then, by summing on $i$ the integrals in all the laminations $\Omega_{1 \mathrm{~s}, \mathrm{i}}$, and averaging the contributions of the subbasis functions products, (7) becomes 


$$
\begin{aligned}
& \left(\mu^{-1} \text { curla,curla' }\right)_{\Omega_{\mathrm{ls}}}=\sum_{\mathrm{m}} \sum_{\mathrm{n}}\left(\overline{\mathrm{N}}_{\mathrm{mn}} \overline{\mathbf{b}}_{\mathrm{n}}, \overline{\mathbf{b}}_{\mathrm{m}}{ }^{\prime}\right) \bar{\Omega}_{\mathrm{ls}} \\
& =\sum_{\mathrm{m}} \sum_{\mathrm{n}}\left(\overline{\mathrm{N}}_{\mathrm{mn}} \operatorname{curl}_{\mathrm{n}}, \overline{\mathbf{a}}_{\mathrm{n}}, \text { curla } \overline{\mathbf{a}}_{\mathrm{m}}{ }^{\prime}\right) \bar{\Omega}_{\mathrm{ls}}
\end{aligned}
$$

with coefficients $\overline{\mathrm{N}}_{\mathrm{mn}}$ of a matrix $\overline{\mathbf{N}}$ defined by

$$
\overline{\mathrm{N}}_{\mathrm{mn}}=\mathrm{d}^{-1} \int_{-\mathrm{d} / 2}^{\mathrm{d} / 2} \mu^{-1} \eta_{\mathrm{m}}(\gamma) \eta_{\mathrm{n}}(\gamma) \mathrm{d} \gamma, \forall \mathrm{m}, \mathrm{n} \in \mathrm{S},
$$

where an upper bar symbol denotes an homogenized entity along the lamination or stack thickness. The coefficients $b_{n}$ of expansion (6) for each lamination are indeed transformed to a continuum $\overline{\mathbf{b}}_{\mathrm{n}}$ when all the laminations are considered together, i.e. through the integration in $\bar{\Omega}_{\mathrm{ls}}$, defining the homogenized laminated region.

The homogenized magnetic flux density coefficients $\overline{\mathbf{b}}_{\mathrm{n}}$ can be expressed as the curl of magnetic vector potentials $\overline{\mathbf{a}}_{\mathrm{n}}$, considered as homogenized as well. In this way, the conservation of the magnetic flux density, i.e., $\operatorname{div} \mathbf{b}=0$, is satisfied for the whole expansion (6), as will be shown. At the discrete level, this vector potential is approximated with edge FEs. Particular definition of sub-basis functions $\eta_{n}(\gamma)$ will be shown to conveniently allow the transposition of the flux conservation.

The fourth term of (3) is developed analogously, this time with primitives of $\eta_{n}(\gamma)$, i.e.,

$$
\begin{aligned}
& -\left(\mathbf{j}_{\mathrm{s}}, \mathbf{a}^{\prime}\right)_{\Omega_{\mathrm{ls}}}=-\sum_{\mathrm{i}}\left(\mathbf{j}_{\beta}, \mathbf{a}^{\prime}\right)_{\Omega_{\mathrm{ls}, \mathrm{i}}} \\
& =-\sum_{\mathrm{i}}\left(\sigma \mathbf{i}_{\gamma} \times \partial_{\mathrm{t}} \int \mathbf{b}_{\alpha} \mathrm{d} \gamma,-\mathbf{i}_{\gamma} \times \int \mathbf{b}_{\alpha}{ }^{\prime} \mathrm{d} \gamma\right)_{\Omega_{\mathrm{ls}, \mathrm{i}}} \\
& =\sum_{\mathrm{i}}\left(\sigma \partial_{\mathrm{t}} \int \mathbf{b}_{\alpha} \mathrm{d} \gamma, \int \mathbf{b}_{\alpha}{ }^{\prime} \mathrm{d} \gamma\right)_{\Omega_{\mathrm{ls}, \mathrm{i}}} \\
& =\sum_{\mathrm{i}}\left(\sigma \partial_{\mathrm{t}} \int \sum_{\mathrm{n}} \mathrm{b}_{\mathrm{n}} \eta_{\mathrm{n}} \mathrm{d} \gamma, \int \sum_{\mathrm{m}} \mathrm{b}_{\mathrm{m}}{ }^{\prime} \eta_{\mathrm{m}} \mathrm{d} \gamma\right)_{\Omega_{\mathrm{ls}, \mathrm{i}}} \\
& =\sum_{\mathrm{i}}\left(\sigma \partial_{\mathrm{t}} \sum_{\mathrm{n}} \mathrm{b}_{\mathrm{n}} \int \eta_{\mathrm{n}} \mathrm{d} \gamma, \sum_{\mathrm{m}} \mathrm{b}_{\mathrm{m}}{ }^{\prime} \int \eta_{\mathrm{m}} \mathrm{d} \gamma\right)_{\Omega_{\mathrm{ls}, \mathrm{i}}} .
\end{aligned}
$$

Then, by summing on $i$ the integrals in all the laminations $\Omega_{1 \mathrm{~s}, \mathrm{i}}$, and averaging the contributions of the primitive subbasis functions products, (10) becomes

$$
\begin{aligned}
& -\left(\mathbf{j}_{\mathrm{s}}, \mathbf{a}^{\prime}\right)_{\Omega_{\mathrm{ls}}}=\sum_{\mathrm{m}} \sum_{\mathrm{n}}\left(\overline{\mathrm{P}}_{\mathrm{mn}} \partial_{\mathrm{t}} \overline{\mathbf{b}}_{\mathrm{n}}, \overline{\mathbf{b}}_{\mathrm{m}}{ }^{\prime}\right) \bar{\Omega}_{\mathrm{ls}} \\
& =\sum_{\mathrm{m}} \sum_{\mathrm{n}}\left(\overline{\mathrm{P}}_{\mathrm{mn}} \partial_{\mathrm{t}} \operatorname{curl} \overline{\mathbf{a}}_{\mathrm{n}}, \operatorname{curl} \overline{\mathbf{a}}_{\mathrm{m}}{ }^{\prime}\right) \bar{\Omega}_{\mathrm{ls}}
\end{aligned}
$$

with coefficients $\overline{\mathrm{P}}_{\mathrm{mn}}$ of a matrix $\overline{\mathbf{P}}$ defined by

$$
\overline{\mathrm{P}}_{\mathrm{mn}}=\mathrm{d}^{-1} \int_{-\mathrm{d} / 2}^{\mathrm{d} / 2} \sigma\left(\int \eta_{\mathrm{m}}(\gamma) \mathrm{d} \gamma\right)\left(\int \eta_{\mathrm{n}}(\gamma) \mathrm{d} \gamma\right) \mathrm{d} \gamma, \forall \mathrm{m}, \mathrm{n} \in \mathrm{S}
$$

The magnetic permeability $\mu$ has generally to be kept in integral (9) in order to consider its possible nonlinear nature. For a non-constant permeability, each coefficient $\overline{\mathrm{N}}_{\mathrm{mn}}$ depends on the evaluation point in $\bar{\Omega}_{\mathrm{ls}}$. In this way, the nonlinear behavior is averaged along the lamination thickness through (9).

\section{SKIN EFFECT SUB-BASIS FUNCTIONS}

Examples of polynomial skin effect sub-basis functions $\eta_{\mathrm{n}}(\gamma)$ are developed here. These can be limited to even orders in case no net current flows in the laminations, which gives, for the expansion order set in (6), $\mathrm{S}=\{0,2,4, \ldots\}$.

For convenience, coordinate $\gamma$ is defined as equal to zero at the mid-thickness of each lamination. It can be also associated with a reference coordinate $\gamma_{\mathrm{r}}$, varying from -1 to 1 along the thickness of each lamination, i.e., defined by $\gamma_{\mathrm{r}}=\gamma$ $2 /$ d.

For convenience, the sub-basis functions can be given the property of orthogonality, i.e.,

$$
\int_{-1}^{+1} \eta_{i}\left(\gamma_{r}\right) \eta_{j}\left(\gamma_{r}\right) d \gamma_{r}=0, \text { for } \mathbf{i} \neq \mathbf{j}, \mathbf{i}, \mathbf{j} \in \mathrm{S},
$$

which, in particular, defines a zero average value for each function $\eta_{\mathrm{n}}$ of order $\mathrm{n}$ greater or equal to 1 ; function $\eta_{0}$ is constant and equal to 1 . Consequently, the coefficient $b_{0}$ in (6) is directly the mean magnetic flux density. For linear magnetic materials, this property also gives matrix $\overline{\mathbf{N}}$ a diagonal form.

Even functions $\eta_{\mathrm{n}}\left(\gamma_{\mathrm{r}}\right), \mathrm{n} \in \mathrm{S}=\{0,2,4, \ldots\}$, can then be determined as

$$
\begin{gathered}
\eta_{0}\left(\gamma_{\mathrm{r}}\right)=1, \eta_{2}\left(\gamma_{\mathrm{r}}\right)=\frac{1}{2}\left(3 \gamma_{\mathrm{r}}^{2}-1\right), \\
\eta_{4}\left(\gamma_{\mathrm{r}}\right)=\frac{1}{8}\left(35{\gamma_{\mathrm{r}}}^{4}-30{\gamma_{\mathrm{r}}}^{2}+3\right), \ldots
\end{gathered}
$$

The associated primitive functions $\tau_{n}\left(\gamma_{r}\right)=\int \eta_{n}\left(\gamma_{r}\right) d \gamma_{r}$, $\mathrm{n} \in \mathrm{S}$, are

$$
\begin{gathered}
\tau_{0}\left(\gamma_{\mathrm{r}}\right)=\gamma_{\mathrm{r}}, \tau_{2}\left(\gamma_{\mathrm{r}}\right)=\frac{1}{2}\left(\gamma_{\mathrm{r}}^{3}-\gamma_{\mathrm{r}}\right), \\
\tau_{4}\left(\gamma_{\mathrm{r}}\right)=\frac{1}{8}\left(7{\gamma_{\mathrm{r}}}^{5}-10{\gamma_{\mathrm{r}}}^{3}+3 \gamma_{\mathrm{r}}\right), \ldots
\end{gathered}
$$

The corresponding functions written in terms of $\gamma$ are given by

$$
\eta_{\mathrm{n}}(\gamma)=\eta_{\mathrm{n}}\left(\gamma_{\mathrm{r}}\right), \quad \tau_{\mathrm{n}}(\gamma)=\frac{\mathrm{d}}{2} \tau_{\mathrm{n}}\left(\gamma_{\mathrm{r}}\right)
$$

Functions of additional orders satisfying (13) can be determined without difficulties. They will act in a hierarchical way in the expansion of $\mathbf{b}_{\alpha}$, only adding coefficients in matrices $\overline{\mathbf{N}}$ and $\overline{\mathbf{P}}$, without modifying the already calculated contributions.

For $S=\{0,2,4\}$ and constant $\mu$ and $\sigma$, matrices $\overline{\mathbf{N}}$ and $\overline{\mathbf{P}}$ are (indexes for rows and columns follow those of set $\mathrm{S}$ )

$$
\overline{\mathbf{N}}=\mu^{-1}\left(\begin{array}{ccc}
1 & 0 & 0 \\
0 & 1 / 5 & 0 \\
0 & 0 & 1 / 9
\end{array}\right), \overline{\mathbf{P}}=\sigma \frac{\mathrm{d}^{2}}{4}\left(\begin{array}{ccc}
1 / 3 & -1 / 15 & 0 \\
-1 / 15 & 2 / 105 & -1 / 315 \\
0 & -1 / 315 & 2 / 693
\end{array}\right)
$$

of which the coefficients can be directly used in terms (8) and (11) present in the magnetic vector potential formulation (3).

\section{CONSTRAINTS ON THE HOMOGENIZED MAGNETIC FLUX DENSITY}

As stated before, the conservation of the magnetic flux density $\mathbf{b}$ has to be expressed in a strong way. In case there is no component of $\mathbf{b}$ perpendicular to the laminations, i.e. $\mathbf{b}_{\gamma}=0$ and $\mathbf{b}=\mathbf{b}_{\alpha}$ given by (6), one has, in each lamination $\Omega_{\mathrm{ls}, \mathrm{i}}$,

$$
\operatorname{div} \mathbf{b}_{\alpha}=\operatorname{div}\left(\sum_{\mathrm{n} \in \mathrm{S}} \mathbf{b}_{\mathrm{n}} \eta_{\mathrm{n}}(\gamma)\right)=\sum_{\mathrm{n} \in \mathrm{S}} \operatorname{div} \mathbf{b}_{\mathrm{n}} \eta_{\mathrm{n}}(\gamma)=0 .
$$

Then, thanks to the orthogonality property (13) of the subbasis functions $\eta_{\mathrm{n}}$, (18) implies that

$$
\operatorname{div} \mathbf{b}_{\mathrm{n}}=0, \forall \mathrm{n} \in \mathrm{S},
$$

which holds in any lamination but also for the homogenized coefficients $\overline{\mathbf{b}}_{\mathrm{n}}$, i.e.,

$$
\operatorname{div} \overline{\mathbf{b}}_{\mathrm{n}}=0, \forall \mathrm{n} \in \mathrm{S}
$$

A non-zero perpendicular component $\mathbf{b}_{\gamma}$, with $\mathbf{b}=\mathbf{b}_{\alpha}+\mathbf{b}_{\gamma}$ (or $\mathbf{b}=\overline{\mathbf{b}}_{\alpha}+\mathbf{b}_{\gamma}$ ), only gives a complementary contribution to coefficient $\mathbf{b}_{0}$ (or $\overline{\mathbf{b}}_{0}$ ), all together satisfying 


$$
\operatorname{div}\left(\mathbf{b}_{0}+\mathbf{b}_{\gamma}\right)=0, \text { or } \operatorname{div}\left(\overline{\mathbf{b}}_{0}+\mathbf{b}_{\gamma}\right)=0,
$$

with all the other relations in (19) or $(20)$, for $\mathrm{n} \neq 0$, unchanged.

It should be noted that, in this case, odd order sub-basis functions have to be considered in the expansion of $\mathbf{b}_{\alpha}$. Indeed, the eddy currents induced by time variations of $\mathbf{b}_{\gamma}$ have to be given even order distributions. Relations (20) and (21b) justify that $\overline{\mathbf{b}}_{0}+\mathbf{b}_{\gamma}$ and all the other coefficients $\overline{\mathbf{b}}_{\mathrm{n}}$ can be expressed as the curl of magnetic vector potentials. The restriction of coefficients $\overline{\mathbf{b}}_{\mathrm{n}}, \forall \mathrm{n} \in \mathrm{S}$, in a plane perpendicular to the laminations (with a priori unknown direction $\mathbf{i}_{\alpha}$ ) can be obtained through the use of a tensorial conductivity in (12), with non-zero values only in the directions parallel to each lamination. Both contributions $\overline{\mathbf{b}}_{0}$ and $\mathbf{b}_{\gamma}$ are naturally mixed through the weak formulation.

\section{APPLICATION}

The homogenized formulation has been applied to a test problem consisting of a 3-D stack of rectangular laminations. The laminations are characterized by a constant relative permeability $\mu_{\mathrm{r}}=2000$ and a conductivity $\sigma=10^{7} \mathrm{Sm}^{-1}$. Two values are considered for their thickness: $d=0.3$ and $0.6 \mathrm{~mm}$. The thickness of the stack is $1.8 \mathrm{~mm}$ and its width is $10 \mathrm{~mm}$. It is excited by an enforced magnetomotive force (m.m.f.) either sinusoidal or triangular, giving a flux parallel to the laminations. The frequency of the sinusoidal m.m.f. varies from $50 \mathrm{~Hz}$ up to $50 \mathrm{kHz}$, with associated skin depth from $0.5 \mathrm{~mm}$ to $0.016 \mathrm{~mm}$. The one of the triangular m.m.f. is either $50 \mathrm{~Hz}, 1 \mathrm{kHz}$ or $5 \mathrm{kHz}$.

The homogenized solutions, with polynomials orders $p$ equal to 0 and 2 in (6), are compared with the solution of the direct method considering the stack as a set of insulated massive conductors (Fig. 3 for a sinusoidal m.m.f. and Fig. 4 for a triangular m.m.f.). A very good agreement is obtained for $\mathrm{p}=2$ up to quite a high frequency, while the accuracy is lower for $p=0$. The mesh of the massive conductors is sufficiently fine to take the small skin depth into account for high frequencies (in addition, insulating layers have to be considered between the laminations), while the mesh of the continuum does not need to satisfy this constraint and is therefore coarse for any frequency.

Other comparisons can also be made. The distribution of $\mathbf{b}_{\alpha}$ can be post-computed by (6), using the homogenized coefficients $\overline{\mathbf{b}}_{\mathrm{n}}$ and can be compared to the magnetic flux density obtained with the direct method in massive conductor regions (Fig. 5). A similar comparison can be done for the current density $\mathbf{j}_{\beta}$, post-computed through (5) and (6), (Fig. 6). Such comparisons point out the accuracy obtained in function of the approximation order $p$ of $\mathbf{b}_{\alpha}$ in (6).

\section{CONCLUSIONS}

A time-domain technique has been developed to take the eddy currents in lamination stacks into account with bconform FE formulations. In order to avoid the explicit definition of all laminations, each laminated region is converted into a continuum in which the eddy currents are taken into account thanks to adapted terms in the weak formulations. For that, each lamination stack is considered as a source region carrying predefined current density and magnetic flux density distributions describing the eddy currents and skin effect in each lamination. The current density is expressed in terms of the magnetic flux density, this latter being approximated with sub-basis functions of which the contributions are averaged in the weak formulations.
The developed method appears attractive for directly taking into account the eddy current effects which are particularly significant for high frequency components. The time domain analysis makes possible the consideration of these effects for both nonlinear and transient phenomena.

\section{REFERENCES}

Bertotti, G., Boglietti, A., Chiampi, M., Chiarabaglio, D., Fiorillo, F. and Lazzari, M. (1991), "Calculation of eddy currents and associated losses in electrical steel laminations", IEEE Trans. Magn., Vol. 27, No. 6, pp. 5007-5009.

Dular, P., Gyselinck, J., Geuzaine, C., Sadowski, N. and Bastos, J.P.A. (2003) "Modeling of thin insulating layers with dual 3-D magnetodynamic formulations", IEEE Trans. Magn., Vol. 39, No. 3, pp. 1139-1142.

Gyselinck, J. and Dular, P. (2004), "A time-domain homogenisation technique for laminated iron cores in three-dimensional finite element models", IEEE Trans. Magn., Vol. 40, No. 2, pp. 856-859.

Krähenbühl, L., Dular, P., Zeidan, T. and Buret, F. (2004), "Homogenization of lamination stacks in linear magnetodynamics", IEEE Trans. Magn., Vol. 40, No. 2, pp. 912-915.

Righi, L.A., Sadowski, N., Carlson, R., Bastos, J.P.A. and Batistela, N.J. (2001) "A new approach for iron losses calculation in voltage fed time stepping finite elements", IEEE Trans. Magn., Vol. 37, No. 5, pp. 3353 3356 .

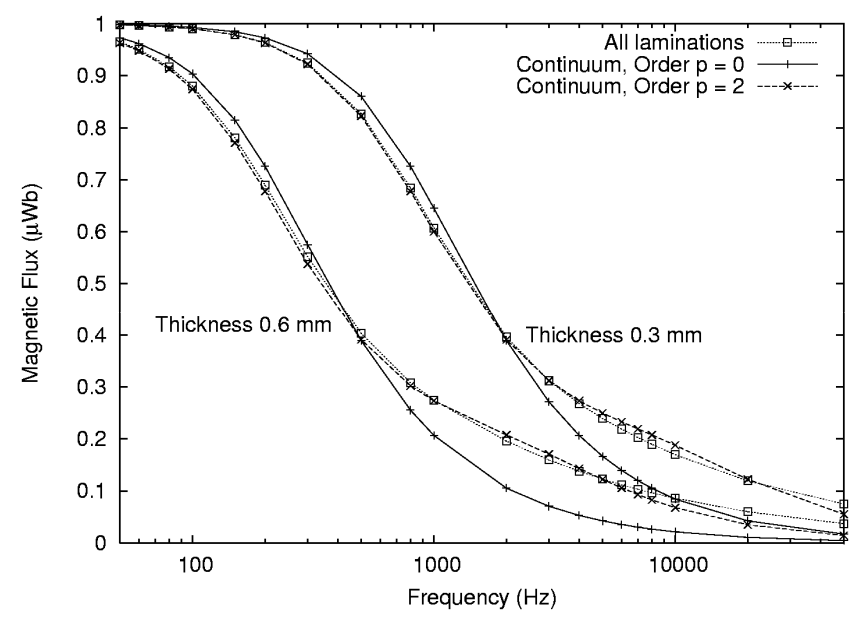

Fig. 3. Amplitude of the magnetic flux in the lamination stack versus the frequency (sinusoidal m.m.f.) with the direct method and the homogenized or continuum model.

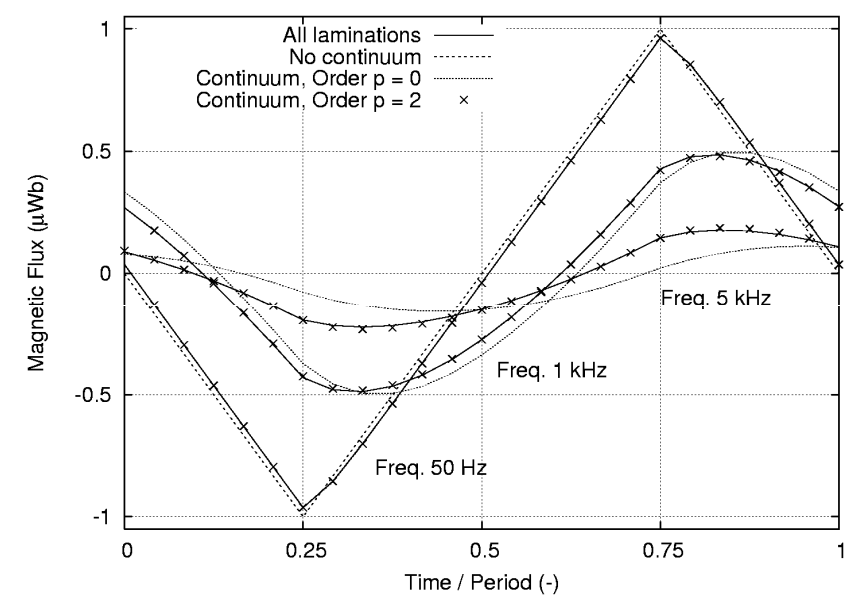

Fig. 4. Magnetic flux versus normalized time with the direct method, no consideration of eddy current (no continuum; a direct image of the m.m.f. is obtained), and the homogenized or continuum model $(\mathrm{d}-0.3 \mathrm{~mm})$; triangular m.m.f. of different frequencies. 


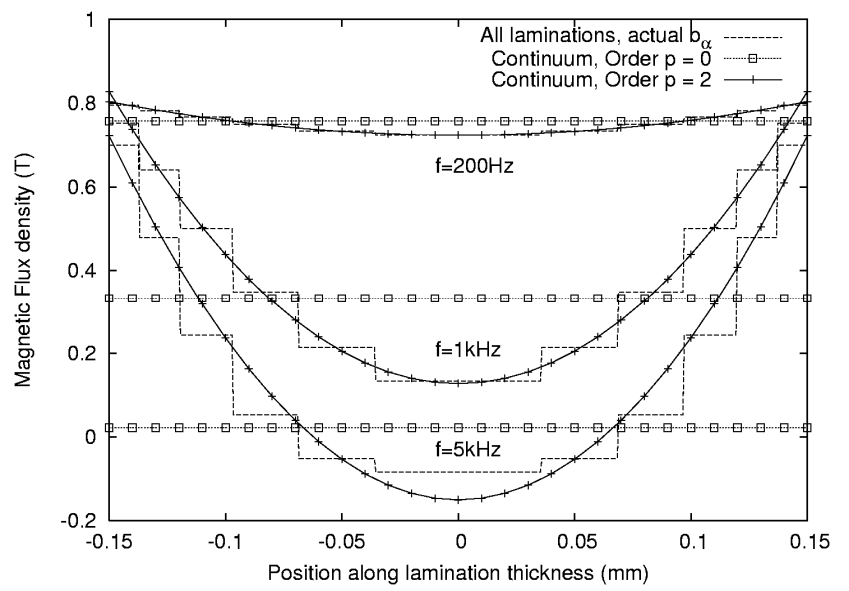

Fig. 5. Magnetic flux density along the thickness of a lamination, at a certain time instant, for different frequencies (sinusoidal m.m.f.) with the direct method and the homogenized or continuum model $(\mathrm{d}-0.3 \mathrm{~mm})$.

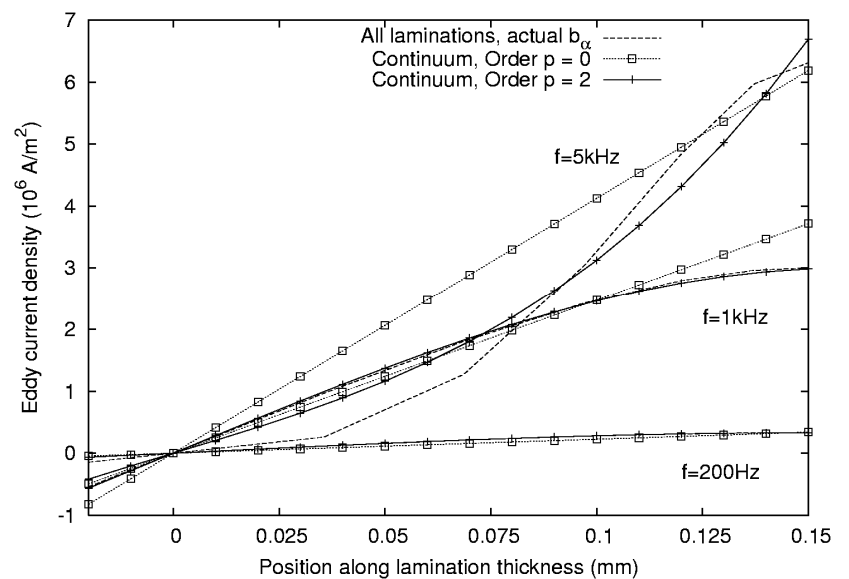

Fig. 6. Eddy current density along the thickness of a lamination, at a certain time instant, for different frequencies (sinusoidal m.m.f.) with the direct method and the homogenized or continuum model $(\mathrm{d}-0.3 \mathrm{~mm})$. 